\title{
Susceptibilidade dos Staphylococcus spp. isolados da pele de cães com piodermite recidivante
}

\author{
Susceptibility of Staphylococcus spp. isolated from the dog's skin with recurrent pyoderma \\ Susceptibilidad de Staphylococcus spp. aislado de la piel de perros con pioderma recurrente
}

Recebido: 08/03/2021 | Revisado: 14/03/2021 | Aceito: 03/04/2021 | Publicado: 13/04/2021

Sinerey Karla Salim Aragão de Sousa
ORCID: https://orcid.org/0000-0002-3994-9358
Universidade Federal Rural da Amazônia, Brasil
E-mail: sinerey@globo.com
Alexandre do Rosário Casseb
ORCID: https://orcid.org/0000-0001-5615-2423
Universidade Federal Rural da Amazônia, Brasil
E-mail:alexcasseb@yahoo.com.br
Elisa Cunha Pereira
ORCID: https://orcid.org/0000-0001-7231-0057
Universidade Federal Rural da Amazônia, Brasil
E-mail: elisa_cunhap@ hotmail.com
Andre Marcelo Conceição Meneses
ORCID: https://orcid.org/0000-0003-3494-2998
Universidade Federal Rural da Amazônia, Brasil
E-mail: andre.meneses1974@icloud.com

\section{Resumo}

Infecções piogênicas mistas acometem todos os mamíferos e todos seus sistemas orgânicos. Portanto, a piodermite também pode ser definida como uma condição infecciosa, produtora de pus, de causa bacteriana que acomete a pele em qualquer nível de profundidade. O presente estudo foi composto por 25 cães portadores de piodermite superficial que tiveram amostras de secreção purulenta coletadas com auxílio de swab, semeadas em meio de cultura para isolamento do Staphylococcus spp., seguida da avaliação da sensibilidade antimicrobiana pelo método de KirbyBauer. A sensibilidade aos antimicrobianos utilizados mostrou que o ciprofloxacino foi a droga que mostrou maior nível de sensibilidade (13/25), enquanto que os mais resistentes foram a Amicacina (19/25), Penicilina (20/25) e a Oxacilina (21/25) respectivamente. A presença de Staphylococcus em processos infecciosos em animais tem se mostrado cada vez mais frequente, e a resistência aos antimicrobianos é uma realidade emergente.

Palavras-chave: Staphylococcus spp; Piodermite recidivante; Cães; Sensibilidade antimicrobiana; Difusão em disco.

\begin{abstract}
Mixed pyogenic infections affect all mammals and their organic systems. Therefore, pyoderma may also be defined as an infectious, pus-producing bacterial condition that affects the skin at any depth level. The present study was composed of $25 \mathrm{dogs}$ with superficial pyoderma that had samples of purulent secretion collected with swab, seeded in culture medium for isolation of Staphylococcus spp. for further evaluation of the antimicrobial susceptibility by the Kirby-Bauer method. Sensitivity to antimicrobials used showed that ciprofloxacin was the drug that showed the highest level of sensitivity (13/25), while the most resistant were Amikacin (19/25), Penicillin (20/25) and Oxacillin (21/25) respectively. The presence of Staphylococcus in infectious processes in animals has been increasingly frequent, and antimicrobial resistance is emerging.
\end{abstract}

Keywords: Staphylococcus spp; Recurrent pyoderma; Dogs; Antimicrobial sensitivity; Disc diffusion.

\section{Resumen}

Las infecciones piógenas mixtas afectan a todos los mamíferos y todos sus sistemas orgánicos. Por lo tanto, la pioderma también se puede definir como una condición infecciosa, productora de pus, de causa bacteriana que afecta la piel a cualquier nivel de profundidad. El presente estudio consistió en 25 perros con pioderma superficial a los que se les tomaron muestras de secreción purulenta con ayuda de hisopo, sembrado en medio de cultivo para aislamiento de Staphylococcus spp., seguido de la evaluación de la sensibilidad a los antimicrobianos por el método de KirbyBauer. La sensibilidad a los antimicrobianos utilizados mostró que el ciprofloxacino fue el fármaco que mostró mayor nivel de sensibilidad (13/25), mientras que los más resistentes fueron Amikacina (19/25), Penicilina (20/25) y Oxacilina (21/25) respectivamente. Se ha demostrado que la presencia de Staphylococcus en procesos infecciosos en animales es cada vez más frecuente, y la resistencia a los antimicrobianos es una realidad emergente.

Palabras clave: Staphylococcus spp; Pioderma recurrente; Perros; Sensibilidad antimicrobiana; Difusión en disco. 


\section{Introdução}

Staphylococcus são bactérias gram-positivas, anaeróbias facultativas e esféricas que normalmente compõem a microbiota da pele íntegra e das mucosas de mamíferos e aves (Griffeth et al, 2008).

A piodermite bacteriana superficial é uma das dermatopatias mais frequentes em cães e trata-se de uma infecção dos folículos pilosos e epiderme adjacente que é na grande maioria das vezes secundária a uma causa de base (Miller, Griffin \& Campbell, 2013). As principais razões para preocupação são, em ordem de importância, S.aureus resistente a meticilina (MRSA) e S. pseudointermedius resistente a meticilina (MRSI) (Guardabassi et al, 2010).

$\mathrm{O}$ termo resistente se refere a aqueles microrganismos que não se inibem pelas concentrações habitualmente alcançadas no sangue ou tecidos do correspondente antimicrobiano, ou aqueles que apresentam mecanismos de resistência específicos para o agente estudado ao qual não havia uma adequada resposta clínica quando usado como tratamento (Rodrigues, et al., 2000; Mota et al, 2005).

A resistência antimicrobiana pode ser de origem genética ou fenotípica (cromossômica e extra cromossômica). Na de origem fenotípica bactéria adquire resistência a determinada droga, momentaneamente e, não consegue transmiti-la para sua progênie. Este mecanismo de origem não genética, geralmente está relacionado a processos de multiplicação bacteriana necessários para a maioria das ações antibacteriana das drogas. O principal mecanismo de uma bactéria sensível tornar-se resistente é através da mutação cromossômica. Outro tipo de resistência pode ser transferida de uma bactéria resistente para uma sensível por contato (Jawetz, et al. 1991; Mota et al, 2005).

Estes agentes apresentam resistência a todas as penicilinas e cefalosporinas, essas bactérias são, com frequência, resistentes a fluoroquinolonas, aminoglicosídeos, tetraciclinas, macrolídeos e lincosaminas (Guardabassi et al, 2010). Os antibióticos são fármacos que revolucionaram o tratamento de doenças infecciosas causadas por bactérias e reduziram mundialmente as taxas de morbidade e mortalidade associadas a infecções bacterianas. Entretanto, o mau uso desses fármacos acelera o processo natural de resistência das bactérias contra os antibióticos, devido ao fato de que no ambiente natural esses antimicrobianos são produzidos por populações microbianas como ferramenta de competição por recursos nutricionais e espaço dentro do micro-habitat que ocupam (Da Costa. \& Junior, 2017).

O problema da resistência antimicrobiana foi extensivamente revisado pelo O'Neill comitê no Reino Unido, que publicou seu relatório final e recomendações em maio de 2016. Após avaliar o problema em escala mundial, puderam concluir que mortes humanas como consequência da resistência antimicrobiana e da incapacidade de tratar e controlar infecções microbianas já eram da ordem de 700.000 por ano e estimado em 10 milhões por ano (um número que inclui infecções com HIV resistente e malária, bem como tuberculose e outras infecções bacterianas), com um custo cumulativo para a produtividade global de US $\$ 100$ trilhões até o ano de 2050, a menos que ações apropriadas sejam tomadas para lidar com este problema (O’neill J, 2016; Lloyd \& Page, 2018).

$\mathrm{O}$ uso de antimicrobianos, principalmente, pelos clínicos de pequenos animais tem na piodermite uma razão frequente para prescrição, e tanto faz se é superficial ou profunda, pois o Staphylococcus pseudintermedius está presente, e em muitos casos recorrentes, temos a presença de S. aureus e S. schleiferi (Pianta et al, 2006; Guardabassi et al, 2010). Em cães saudáveis, S. pseudintermedius faz parte da flora cutânea normal, colonizando pele, folículo piloso, revestimento, em particular, sítios mucocutâneos, como o nariz, boca e ânus. Este constitui cerca de 90\% dos Staphylococcus spp. isolados de cães saudáveis e com algum problema de pele subjacente (Allaker, et al., 1992; Fazakerley, 2009; Bannoehr, Guardabassiz, 2012).

Portanto, o presente estudo teve como objetivo isolar Staphylococcus spp. de cães com piodermite e avaliar a sua sensibilidade frente aos antimicrobianos, com o objetivo de identificar quais bases de antibióticos mais utilizados na Medicina Veterinária apresentam resistência. 


\section{Metodologia}

O presente projeto foi aprovado pelo comité de ética da UFRA (CEUA-UFRA) sob o Protocolo 004/2014 (CEUA) 23084.001959/2014-99 (UFRA).

A população de estudo foi composta por 25 cães com piodermite superficial recidivante tendo como causa de base a dermatite atópica canina (DAC), que é uma condição que dentre várias alterações, causa diminuição da barreira de proteção cutânea.

As amostras de secreção purulenta foram coletadas diretamente das lesões desses animais com auxílio de swab e armazenados em meio de Stuart e posteriormente cultivadas.

O material foi semeado em meio de Ágar sangue de carneiro a 5\%, Ágar Mackonkey, caldo cérebro-coração e incubadas a $37^{\circ} \mathrm{C}$, em aerobiose. A espécie microbiana foi identificada com base na morfologia de colônia, coloração pelo método de Gram e provas bioquímicas (Ikram, 2005). Para realização dos testes de sensibilidade dos micro-organismos a fármacos antimicrobianos, utilizou-se o método de Kirby-Bauer (Bauer, 1966), usando os seguintes discos de antibióticos: Amoxicilina + Ácido clavulânico (AMC), Amicacina (AMI), Ampicilina + Sulbactam (ASB), Ciprofloxacino (CIP), Cefalotina (CFL), Cloranfenicol (CLO), Gentamicina (GEN), Nitrofurantoína (NIT), Norfloxacino (NOR), Oxaciclina (OXA), Penicilina (PEN), Rifampicina (RIF), que foram disponibilizados em duas placas e para esta análise, fez-se a leitura dos resultados com base no halo formado por cada antibiótico.

\section{Resultados}

Dentre os resultados encontrados, a sensibilidade antimicrobiana do Staphylococcus spp isoladas (Tabela 1), observou-se que houve maior expressão de resistência bacteriana do que sensibilidade, dentre as bases que apresentaram maior resistência estão a Oxacilina, seguidos de Nitrofurantoina e Penicilina. Enquanto apenas a Ciprofloxacina apresentou maior sensibilidade, apesar de pouco expressiva quando comparada com a porcentagem de resistência.

Tabela 1 - Resultados obtidos de sensibilidade e resistência a antimicrobianos de Staphylococcus spp isolodo de piodermatite de cães atendidos no Hovet/UFRA.

\begin{tabular}{l|c|c|c}
\hline Antimicrobiano & Sensivel & Intermediaria & Resistente \\
\hline Amoxicilina + Ac. Clavulânico (AMC) & $11(44 \%)$ & ------------------ & $14(56 \%)$ \\
\hline Amicacina (AMI) & $05(20 \%)$ & $01(4 \%)$ & $19(76 \%)$ \\
\hline Ampicilina + Sulbactan (ASB) & $06(24 \%)$ & $02(8 \%)$ & $18(72 \%)$ \\
\hline Ciprofloxacino (CIP) & $13(52 \%)$ & $01(4 \%)$ & $11(44 \%)$ \\
\hline Cefalotina (CFL) & $08(32 \%)$ & -------------------- & $17(68 \%)$ \\
\hline Cloranfenicol (CLO) & $08(32 \%)$ & ------------------- & $17(68 \%)$ \\
\hline Gentamicina (GEN) & $10(40 \%)$ & $01(4 \%)$ & $14(56 \%)$ \\
\hline Nitrofurantoina (NIT) & $05(20 \%)$ & ------------------ & $20(80 \%)$ \\
\hline Norfloxacino (NOR) & $11(44 \%)$ & ------------------- & $14(56 \%)$ \\
\hline Oxacilina (OXA) & $04(16 \%)$ & ----------------- & $21(84 \%)$ \\
\hline Penicilina (PEN) & $05(20 \%)$ & ------------------ & $20(80 \%)$ \\
\hline Rifampicina (RIF) & $08(32 \%)$ & -------------------- & $17(68 \%)$ \\
\hline
\end{tabular}

Fonte: Autores. 


\section{Discussão}

A análise aos antimicrobianos utilizados no teste de sensibilidade mostrou que a Ciprofloxacino foi a droga com maior nível de sensibilidade (13/25), enquanto que os mais resistentes foram a Amicacina (19/25), Nitrofurantoina (20/25), Penicilina (20/25) e a Oxacilina (21/25) respectivamente. Tunon et al (2008) isolaram cepas de Staphylococcus spp. em otites caninas e encontraram 83\% de cepas resistentes a pelo menos um dos antibióticos utilizados, enquanto que Pianta et al (2006) encontraram cerca de 95\% de sensibilidade a Amoxicilina com Ácido Clavulânico de piodermas caninos por eles examinados.

Apesar da Ciprofloxacina ter sido a droga de maior sensibilidade neste estudo, não houve diferença significativa em relação a sua resistência. A grande maioria se apresentou mais resistência aos antibióticos testados. Este resultado difere do encontrado por Carvalho (2017) em Staphylococcus pseudointermedius isolados de piodermatite de cães e gatos atendidos na Universidade de Londrina, observou-se 67\% de sensibilidade a Ciprofloxacina, enquanto que para outros antibióticos como Gentamicina, Amoxicilina com Clavulanato de Potássio, Ciprofloxacina e Cefalexina foram 85\%, $72 \%$ e 56\% de sensibilidade, respectivamente. Eles também observaram que os antibióticos Penicilina G, Neomicina, Sulfazotrin e Enrofloxacina apresentaram 100\%, 66\%, 45\% e 40\% de resistência, respectivamente.

De acordo com Penteado Filho et al (2007) os testes de sensibilidade convencionais, como o método de difusão em disco, nem sempre são sensíveis o suficiente para detectar populações heterorresistentes. Atualmente, o teste de triagem para essas cepas é feito por meio da verificação da sensibilidade à Oxacilina e à determinação da presença do gene mecA por técnicas moleculares utilizando a reação em cadeia mediada pela polimerase (PCR). A resistência a Oxacilina detectada neste estudo pelo método Kirby-Bauer foi de 21/25 (84\%).

Uma importante fonte de resistência em bactérias é o uso de antimicrobianos na agricultura e medicina veterinária, devido ao aumento da demanda global por proteína animal para consumo humano (carne e leite). Embora o uso de antimicrobianos na prática de animais de companhia é muito menor do que em animais de fazenda, a relação próxima entre animais tratados, equipe veterinária, proprietários e suas famílias, e o público aumentou o risco de transferência e infecção entre eles (Van Boeckel, et al. 2015; Damborg, et al. 2016; Lloyd \& Page, 2018). O aparecimento de resistência bacteriana em animais de companhia não tem recebido a devida importância, quando se leva em consideração a saúde humana e animal, afinal os pets estão cada vez mais compondo ambientes familiares nos lares ao redor do mundo, e isso é um risco potencial a saúde dessas espécies. Ainda, vale ressaltar que a transferência de microrganismos resistentes ocorre não apenas de animais para humanos, mas também na outra direção, como evidenciado pela ocorrência de colonização e infecção hospitalar por Staphylococcus aureus resistente à meticilina (MRSA) em cães e gatos (Soares, et al, 2010; Fritz, et al. 2014; Lloyd \& Page, 2018). Como defendido por alguns autores estão surgindo, do ponto de vista clinico, resistência bacteriana, principalmente de cães (Guardabassi, et at, 2004). As principais razões para preocupação são, em ordem de importância nos cães o $S$. pseudointermedius resistente a Meticilina (MRSP) e S.aureus resistente a Meticilina (MRSA) (Guardabassi et al, 2010).

O S. aureus está presente em uma grande variedade de infecções sendo o tratamento indicado o uso de antibióticos, esse agente, como demonstrado e discutido neste trabalho, é capaz de tornar-se resistente a todas as classes de antibióticos. Seja através de mutações em genes cromossomais ou através da aquisição da transferência de determinantes horizontais de resistência (Vestergaard, 2019).

\section{Conclusão}

A presença de Staphylococcus spp. em processos infecciosos em animais é uma realidade comprovada em estudos anteriores, a resistência aos antimicrobianos testados nesse trabalho foi bastante significativa, principalmente devido ao uso de antibióticos, algumas vezes sem critérios, por parte de alguns profissionais que atuam na área de pequenos animais.

A multirresistência bacteriana aos antimicrobianos deve ser encarada como um problema de Saúde Pública emergente. 
Na Medicina Veterinária deve ser mais bem avaliada e estudada, pois diante do aumento do número de casos de multirresistência, principalmente devido o aumento de cães intradomiciliados.

Devemos lembrar que as bactérias multirresistentes já são um problema em grandes centros urbanos, por isso o seu controle é uma necessidade indiscutível.

A cultura com antibiograma deve ser adotada para a detecção do agente bacteriano e a escolha do antibiótico de melhor resposta. O método Kirby-Bauer mostrou ser eficaz na constatação da sensibilidade e resistência aos antimicrobianos.

\section{Referências}

Allaker, R. P., Lloyd, D. H., \& Simpson, A. I. (1992) Occurrence of Staphylococcus intermedius on the hair and skin of normal dogs. Res Vet Sci. 52, 174176. $10.1016 / 0034-5288(92) 90006-n$

Bauer, A. W., Kirb, W. M. M., Sherris, J. C. et al. (1966). Antibiotics susceptibility testing by a standardized single disk method. American Journal of Pathology, 19, 493-496. https://doi.org/10.1093/ajcp/45.4_ts.493

Bannoehr, J., \& Guardabassiz, L. (2012). Staphylococcus pseudintermedius in the dog: taxonomy, diagnostics, ecology, epidemiology and pathogenicity. Vet Dermatol. 23(4):253-e52. 10.1111/j.1365-3164.2012.01046.x

Biberstein, E. L., Hirsh, D. C. In: Hirsh, D. C., \& Zee, Y. C. (2003). Microbiologia Veterinária. Guanabara Koogan. (2a ed.), $108-112$.

Carvalho, C. A. (2017). Piodermatite em cães:aspectos revisionais e contribuição clinica prospectiva. Dissertação de Mestrado. Paraná.

Da Costa, A. L. P., \& Junior, A. C. S. S. (2017). Resistência bacteriana aos antibióticos e Saúde Pública: uma breve revisão de literatura. Estação Científica (UNIFAP), 7(2), 45-57. http://dx.doi.org/10.18468/estcien.2017v7n2.p45-57

Damborg, P. et al. (2016). Bacterial zoonoses transmitted by household pets: state-of-the-art and future perspectives for targeted research and policy actions. $J$ Comp Pathol, 155, S27-S40. http://dx.doi.org/10.1016/j.jcpa.2015.03 .004.

Fazakerley, J., Nuttall, T., Sales, D. et al. (2009). Staphylococcal colonization of mucosal and lesional skin sites in atopic and healthy dogs. Vet Dermatol. 20, $179-184$.

Fritz, S. A. et al. (2014). Contamination of environmental surfaces with Staphylococcus aureus in households with children infected with methicillin-resistant S aureus. JAMA Pediatr, 168, 1030-1038. http://dx.doi.org/10.1001/jamapediatrics.2014.1218.

Griffeth, G. C. et al. (2008). Screening for skin carriage of methicillin-resistant coagulase positive staphylococci and Staphylococcus schleiferi in dogs healthy and inflamed skin. Veterinary Dermatology. 19(3), 142-149. https://doi.org/10.1111/j.1365-3164.2008.00663.x

Guardabassi, L., Schwarz, S., \& Lloyd, D. H. (2004). Pet animals as reservoirs of antimicrobial-resistant bacteria. Journal of Antimicrobial Chemotherapy. 54 (2), 321-322.

Guardabassi, L. et al. (2010). Orientações para o uso de antimicrobianos em cães e gatos. In: Guardabassi, L., Jensen, L. B., Kruse, H. Guia de antimicrobianos em veterinária. Artmed. 224-246.

Jawetz, E. et al. (1991). Microbiologia médica. Guanabara Koogan, ed. 15, 519.

Ikram, M. (2013). Microbiologia diagnóstica. In: Hendrix, C. M. Procedimentos laboratoriais para técnicos veterinários. São Paulo: Roca. 4. ed., 109-225.

Lloyd, D. H., \& Page, S. W. (2018). Antimicrobial Stewardship in Veterinary Medicine.Microbiology spectrum,6 (3). https://doi.org/10.1128/microbiolspec.ARBA-0023-2017.

Miller, W. H., Griffin, C. E. \& Campbell, K. L. (2013). Atopic disease. In: Muller and Kirk's Small Animal Dermatology. Saunders-Elsevier. (7a ed.), 364388 .

Mota. R. Aç et al. (2005). Utilização indiscriminada de antimicrobianos e sua contribuição a multirresitência bacteriana. Braz J vet Res anim Sci. 42 (6), 465 470. 10.11606/issn.1678-4456.bjvras.2005.26406.

O'Neill J (chair) (2016). The review on antimicrobial resistance. Tackling drug resistant infections globally: final report and recommendations. AMR Review Paper - Tackling a crisis for the health and wealth of nations_1.pdf (amr-review.org)>.

Penteado Filho, S. R., Lopes, H. V., \& Levi, G. C. (2007). Antibióticos clássicos: principais características e uso terapêutico. In: Neto, V. A., Nicodemo, A. C., Lopes, H. V. Antibióticos na Prática Médica. Sarvier. (6a ed.), 94-96.

Pianta C. et al. (2006). Pioderma estafilocócico canino: identificação das espécies e sensibilidade aos antimicrobianos. Revista de Ciências Agroveterinárias, 5 (1), 60-63.

Rodriguez, J. A. G. et al. (2000). Procedimientos em microbiología clínica. Métodos básicos para el estudio de la sensibilidad a los antimicrobianos. < seimc-procedimientomicrobiologia11.pdf $>$.

Soares, M. R. J. et al. (2010). Risk factors for methicillin-resistant Staphylococcus aureus (MRSA) infection in dogs and cats: a case-control study. Vet Res, 41 (55). http://dx.doi.org/10.1051/vetres/2010028. 
Research, Society and Development, v. 10, n. 4, e34910413648, 2021

(CC BY 4.0) | ISSN 2525-3409 | DOI: http://dx.doi.org/10.33448/rsd-v10i4.13648

Tunon, G. I. L., Silva, E. P., \& Faierstein, C. C. (2008). Isolamento de Estafilococos Multirresistentes de Otites em Cães e sua Importância para a Saúde Pública. Boletim Epidemiológico Paulista, 58 (5).

Van B. T. P. et al. (2015). Global trends in antimicrobial use in food animals. Proc Natl Acad Sci USA, 112, 5649-5654. http://dx.doi.org/10.1073/pnas.1503141112.

Vestergaard, M., Frees, D., \& Ingmer, H. (2019). Antibiotic Resistance and the MRSA Problem. Microbiology spectrum,7(2), https://doi.org/10.1128/microbiolspec.GPP3-0057-2018 